\title{
Is the Madden-Julian Oscillation reliably detectable in Schumann Resonances?
}

\author{
Ciarán D. Beggan*, Malgorzata A. Musur ${ }^{1}$ \\ British Geological Survey, Riccarton, Edinburgh, EH14 44AP, UK.
}

\begin{abstract}
The Madden-Julian Oscillation (MJO) is a quasi-periodic ( $\sim 30-90$ days) eastwardmoving atmospheric mode which primarily modifies rainfall patterns in the equatorial regions from Africa to the Pacific Ocean. It has been proposed that its signature is detectable within the intensity variations of the Schumann Resonances (SR) due to changes in the location and magnitude of the major lightning centres. Using six years of induction coil data recorded at the Eskdalemuir Observatory in the UK, we investigate whether the MJO is detectable in the first three Schumann Resonances. We extract the frequency and intensity values from each resonance every 10 minutes, averaged to a daily value and compare them to the Realtime Multivariate MJO (RMM) index. We use Empirical Mode Decomposition (EMD) to determine if certain modes correlate between the SR and RMM curves. We find that the EMD 30 to 70 day modes of the SR and RMM index occasionally beat in-phase during the La Niña periods of the El Niño Southern Oscillation (ENSO) but not El Niño periods. However, the relationship is not wholly consistent, implying that robust and reliable detection of the MJO in SR data remains challenging.

Keywords: Magnetic field, Schumann Resonances, Madden-Julian Oscillation, Southern Oscillation Index, ENSO
\end{abstract}

\footnotetext{
${ }^{*}$ Corresponding author

Email address: ciar@bgs.ac.uk (Ciarán D. Beggan)

${ }^{1}$ Visiting researcher
}

Preprint submitted to Journal of Atmospheric and Solar-Terrestrial Physics April 24, 2019 


\section{Introduction}

Since their theoretical prediction by Schumann (1952) and experimentally detection by Balser \& Wagner (1960), the Schumann Resonances (SR) have been widely studied. Schumann Resonances are electromagnetic (EM) oscillations

5 with wavelengths approximately divisible by the Earth's circumference that are trapped within the Earth-ionosphere cavity. They are excited by the nearcontinuous global lightning discharge forming the EM background. The SR are observed below $50 \mathrm{~Hz}$ in the Extremely Low Frequency (ELF) band of the electromagnetic spectrum and the first five resonances are usually found at approximately 7.8, 13.9, 20.1, 26.5 and $32.4 \mathrm{~Hz}$ (e.g. Füllekrug, 1995; Price 2016) though with a low Q-factor, typically less than 4 (e.g. Nickolaenko \& Hayakawa, 2002, Sentman, 1995).

In the past five decades, research has revealed information about the manner of their propogration within the Earth-ionosphere cavity, their response to the solar cycle and their use as a monitor (or proxy) for other geophysical systems and atmospheric modes (e.g. Anyamba et al., 2000, De et al., 2010, Greenberg \& Price, 2007, Kułak et al., 2003; Nickolaenko et al., 2015, Ondrášková et al. 2011; Satori et al. 1996, Williams, 1992). The SR have been used as a proxy for global lightning activity; the correlation between lightning activity and the diurnal variation in the SR frequencies and intensity is well established (e.g. Will, 2005, Nieckarz et al., 2009). Most lightning activity occurs around the equatorial regions, created by large convective clouds formed in the local afternoon. Diurnal variations are easily seen in the SR spectral parameters (that is, amplitude/intensity, peak frequency, Q-factor) and have three main peaks in 25 intensity at 0800, 1400 and 2000 UT correlated with the crossing of the subsolar point over the main continental regions of Asia, Africa and America (e.g. Greenberg \& Price, 2007, Ondráśková et al., 2007; Sátori, 1996).

The amplitude of SR are influenced by the source intensity and sourceobserver distance as well as the properties of the cavity through which the EM waves propagate. Williams \& Sátori (2007) noted the variations of the 
SR waveguide height over a range of timescales including diurnal, 27-day solar rotation cycle, annual and the 11-year solar cycle. The diurnal and seasonal variations in peak frequency of the first four SR modes (i.e. 7.8, 13.9, 20.1 and $26.5 \mathrm{~Hz}$ ) for the electric $\left(\mathrm{E}_{z}\right)$ field component were analysed by Ondrás̆ková

35 et al. (2007) and were ascribed to changes in the position of the lightning sources as well as changes in the ionosphere height-conductivity profile. Füllekrug \& Fraser-Smith (1996) analysed the amplitudes of the first two SR modes and found 20-30 day variations that they related to the solar rotation cycle (based on comparisons with the sunspot number).

Zieger \& Sátori (1999) compared long-term periodic variations in SR with solar wind speed and geomagnetic indices (Kp and Dst). Their study found a number of periodicities including 9-10, 13.5, 20, 27, 47, 73 and 108 days with the most significant finding relating to the correlation of half solar-rotation period (13.5 day variation) within the SR. While looking into monitoring global thun45 derstorm activity, Nickolaenko et al. (2015) compared 10 years (2002 - 2012) of average daily frequency and intensity data of the first SR mode measured at the Antarctic station in Vernadsky with corresponding solar activity (intensity of solar radiation at the $10.7 \mathrm{~cm}$ wavelength and the Wolf's number). The results show that the SR parameters follow the overall 11-year solar cycle trend. Sim50 ilarly, analysis of the solar-related variation by Sátori et al. (2005) and Kułak et al. (2003) found that the Earth-ionosphere cavity undergoes long-term variation during the 11-year solar cycle due to changes in the conductivity from enhanced X-ray emissions.

On ENSO (El Niño Southern Oscillation) timescales, a north-south shift of global lightning was identified by Sátori \& Zieger (1999) based on SR frequency variations and semiannual intensity changes at Nagycenk, in Hungary. The global lightning shifts southward in the warmer El Niñao period and migrates northward in cooler La Niña episode by around $5^{\circ}$ in latitude. Redistribution of global lightning on the ENSO time scale was confirmed by independent lightning observations by OTD/LIS satellites (Sátori et al., 2009). Global lightning activity over land increases slightly, mainly in South-East Asia in El Niño pe- 
riods, while the lightning activity in the Pacific region significantly decreases; although it is only a minor contributor to the total lightning. Conversely, in La Niña periods Pacific lightning intensity rises.

Intensity variations of the SR were analysed by Anyamba et al. (2000) who suggested that changes in the intensity over 30-90 day periods were controlled by meteorological variability in the troposphere, rather than the 27-day solar rotation cycle. To prove their hypothesis, Anyamba et al. (2000) compared their SR periodicities to the modulation of the global deep convection known as the Madden-Julian oscillation (MJO). The MJO is the dominant mode of the tropical atmospheric intraseasonal variability associated with large scale changes in the upper and lower level winds, sea surface temperature, vertical motion and atmospheric moisture content (Zhang, 2005).

Anyamba et al. (2000) suggested that global variations in SR intensities 75 on these periods are modified by the changes in deep convection due to MJO due to the movement of the locations of the lightning centres. However, they based their findings on short (four month) segments of data from Antarctica chosen from seven years of incomplete SR meaurements. They analysed a single frequency $(10 \mathrm{~Hz})$ from four universal times per day: 0200, 0800, 1400 and 2000

so UT and made a somewhat subjective and qualitative comparison to their own Convective Index parameter. This parameter was derived from the analysis of outgoing longwave radiation (OLR) measurements, created as a proxy for the MJO. Their study is commonly cited as proving the SR-MJO relationship, but, to our knowledge, there have been no further repeat studies in the literature to confirm these findings.

In 2012, two horizontal induction coil magnetometers were installed at a geophysical observatory in Eskdalemuir (Scottish Borders, UK) by the British Geological Survey (BGS). The coils have been recording magnetic field variations over an effective frequency range of $0.1-50 \mathrm{~Hz}$ since September 2012 (Beggan \& Musur, 2018). Having collected around six years of induction coil data, the main focus of this study is to re-assess the effects of the MJO in SR, in order to provide a repeat study of the work in Anyamba et al. (2000), us- 
ing a larger, more complete dataset and harnessing the greater contemporary computing resources available.

In Section 2 we describe the instrumentation at Eskdalemuir, the manner of data collection and the methodology used in the study. In Section 3 we analyse the long-term signals in our data. In Section 4, we focus on looking for the relationship between the MJO signals within the SR data as described by Anyamba et al. (2000), analysing the periodicities using the Lomb-Scargle transform and empirical Empirical Mode Decomposition. The results are discussed in Section 5.

\section{Instrumentation}

The BGS Eskdalemuir Geophysical Observatory is sited in a magnetically quiet valley in the Scottish Borders, UK $\left[55.3^{\circ} \mathrm{N},-3.2^{\circ} \mathrm{E}\right]$. The geomagnetic latitude of the observatory (in quasi-dipole coordinates) at 2018.0 was $57.5^{\circ} \mathrm{N}$ $(\mathrm{L}$-shell $=3.46)$.

In June 2012 two refurbished CM11E high-frequency induction coil magnetometers were installed on site. The horizontal coils were installed on levelled gravel beds, set perpendicular to each other, orientated to geographic northsouth and east-west (within $\pm 2^{\circ}$ ). They are covered by a wooden frame to protect them from the weather, and sited at the edge of the observatory grounds in the middle of an open field to reduce anthropogenic interference.

The induction coils measure small and very rapid magnetic field variations in the Extremely Low Frequency (ELF) band range of $0.1-50 \mathrm{~Hz}$. The analog voltage output signal is converted by a 24-bit Guralp digitizer for wired transmission to a logger placed inside a secure vault around $150 \mathrm{~m}$ away. The data are sampled at $100 \mathrm{~Hz}$ by a local computer where they are recorded; each second is time-stamped using GPS. Every 5 minutes, the data are automatically collected and permanently stored on the BGS computer network in Edinburgh. In total, between September 2012 and September 2018 there were around 120 days of data missing due to computer issues or component failure ( $\sim 95 \%$ availability). 
The analysis of the raw data is initially made in the frequency domain using Welch periodograms (Welch, 1967). Dynamic spectrograms are created for each day of data from local midnight (00:00 UT) to midnight the following day. To do this, we use a Butterworth five-pole filter with a $3-40 \mathrm{~Hz}$ bandpass applied to the raw digitizer data. One hundred seconds (10 000 points) are selected from which a series of fast Fourier transforms are computed using the Welch method (with $100 \mathrm{~s}$ of filtered data) to produce 864 1-D spectra plots per day. A Hanning window is applied (overlap of 100 points between windows) to taper the data prior to applying a 4,096-point Fourier transform. Individual periodograms are converted to SI units using the known calibrated instrument response ( 50 $\mathrm{mV} / \mathrm{nT}$ between 0.1 and $100 \mathrm{~Hz}$ for the analog coils), and digitizer calibration values. The digitizer has a fixed conversion factor of $3.491 \mu \mathrm{V} /$ count for the north-south channel (CH1) and $3.475 \mu \mathrm{V} /$ count for the east-west channel (CH2). This gives a digitization level of around $0.07 \mathrm{pT} /$ count in both coils for short-period signals.

Figure 1 shows example spectrograms from January and August 2018 from each coil. The Schumann resonances are visible as diffuse vertical bands at around 8, 14, 20, 26, 32 and $38 \mathrm{~Hz}$. The strong thin line at $25 \mathrm{~Hz}$ is a subharmonic of the UK electrical power system. Horizontal lines are due to missing data or regional lightning strikes (typically within $1000 \mathrm{~km}$ ) which raises the average power in that particular $100 \mathrm{~s}$ time window. The spectrograms are fixed to the same color scale, allowing the differences in intensity between winter and summer periods to be noted. Although the intensity changes are readily observed, there are also subtle variations in the peak frequency value of each resonance over time.

\section{Time-Universal Time analysis of seasonal signals}

Though daily and seasonal variations are visible in the spectrograms in Figure 1. to find changes on inter-seasonal time periods a different approach is 

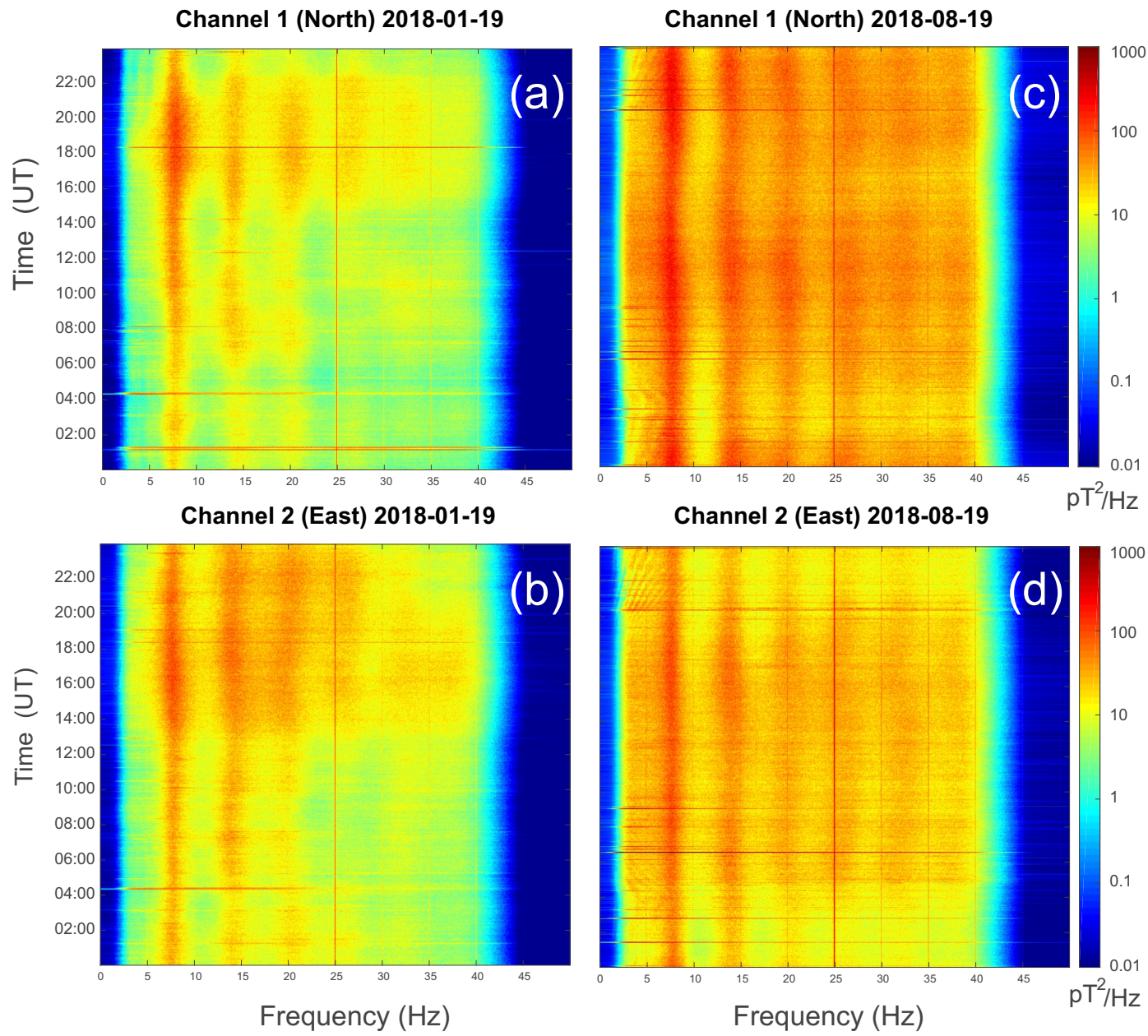

Figure 1: Example spectrograms from the North-South (CH1) and East-West (CH2) orientated coils at Eskdalemuir showing the Schumann Resonances. Note the data are band-pass filtered between 3 and $40 \mathrm{~Hz}$. (a,b) winter:19-Jan-2018 (c,d) summer:19-Aug-2018. 
of Nickolaenko et al. (2015). In reducing the voluminous dataset down to a more compact form, we note there is a trade off between time resolution of the signals, robustness to noise and ease of computation. The study of Anyamba et al. (2000) chose a single frequency at four time points each day due to the limited bandwidth and computing resources available at the time. With larger amounts of computing capacity now available, it is possible to examine the entire SR dataset in more detail.

We examined the intensity and peak frequency properties of the first three resonances using a frequency-domain averaging approach. Following Nickolaenko et al. (2015), the time-domain data for each day were divided into 10minute intervals and Fast Fourier Transformed to give 144 spectra which were converted to SI units using the calibration values noted in Section 2.

We then computed the average intensities and peak frequencies for each SR mode. The peak frequencies $(f)$ for the first SR mode in both coils $\left(f_{S R 1}^{N S}\left(t_{i}\right)\right.$ and $\left.f_{S R 1}^{E W}\left(t_{i}\right)\right)$ were calculated as a weighted average using the following equation, for every 10 -minute interval $t_{i}$ :

$$
f_{S R 1}^{N S, E W}\left(t_{i}\right)=\frac{\int_{f_{0}-\Delta f}^{f_{0}+\Delta f} f \cdot S^{N S, E W}\left(f, t_{i}\right) d f}{\int_{f_{0}-\Delta f}^{f_{0}+\Delta f} S^{N S, E W}\left(f, t_{i}\right) d f},
$$

where $S^{N S, E W}\left(f, t_{i}\right)$ are power spectra calculated for the separate north-south (NS) and east-west (EW) channels. The first SR central frequency used by Nickolaenko et al. (2015) was set to $f_{0}=8.0 \mathrm{~Hz}$ with $\Delta f=1.5 \mathrm{~Hz}$, giving a 170 range of $6.5 \mathrm{~Hz}-9.5 \mathrm{~Hz}$. For the second and third SR, the central frequencies were set at 14 and $21 \mathrm{~Hz}$, respectively, with the same $\Delta f=1.5 \mathrm{~Hz}$.

The SR intensity $(I)$ was calculated from the amplitude of spectral power at the peak frequency:

$$
I_{S R 1}^{N S, E W}\left(t_{i}\right)=S^{N S, E W}\left(f_{S R 1}^{N S, E W}, t_{i}\right)
$$

The data can be visualized in a time-Universal Time (UT) plot for an entire 175 year, with the days of the year on the $\mathrm{y}$-axis and universal time on the $\mathrm{x}$-axis. 
We can further reduce the noise by averaging several years of time-UT plots together. Figure 2 shows the annual-average Time-Universal Time plots using six years of data. The top row (a) shows the intensity of the first Schumann Resonance (SR1) from the north-south coil (left) and the east-west coil (right), while the bottom row (b) shows the peak frequency.

The intensity plot for the north-south coil shows the magnitude increasing around local midday, particularly in summer, as thunderstorm activity begins in India, while the east-west channel has a maximum magnitude around 16:00 related to the African centre. The magnitude of the resonance is greatest in northern hemisphere summer. The sunrise terminator line is faintly visible in these plots, though the sunset line is not as clear (Melnikov et al., 2004 Ondrášková et al. 2007).

The frequency plot for the north-south coil (Fig. 2(b)) illustrates the sourcereceiver distance phenomenon where the highest frequencies (around $8 \mathrm{~Hz}$ ) occur at 06:00 and 20:00, particularly in winter time when the thunderstorm centres are further away from Eskdalemuir. The path length travelled by EM waves to the east-west coil varies throughout the year, being closest in summer (when the Sun reaches the tropic of Cancer) and furthest at the winter solstice. The sunrise terminator is also clearest in this plot.

195 The intensity and frequency plots for the other resonances show various subtleties related to the source receiver distance and orientation (see Supplementary Information). Individual plots for each year are noisier but show similar seasonal patterns. To examine short time-scale variations, the mean annual TimeUT plots were subtracted from the individual years. However, it was difficult to discern if there were any shorter non-periodic signals within the data, due to the background noise. Finally, we note the same method was applied to one year of induction coil data from the Conrad Observatory in Austria and yielded similar seasonal variation patterns in the first three Schumann Resonances (not shown). 

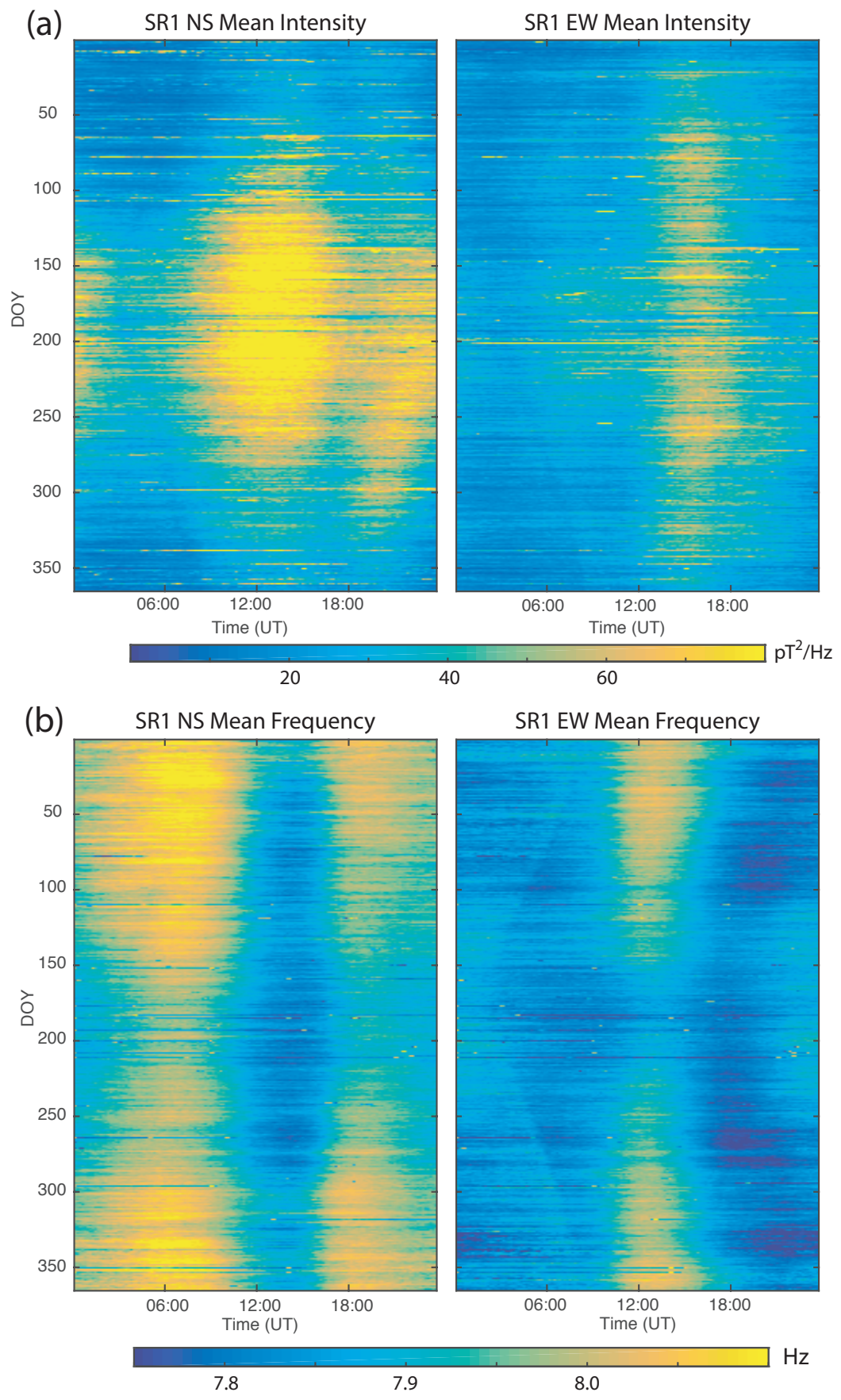

Figure 2: Annual average of six years Time-Universal Time plots of the intensity and the peak frequency of the first Schumann Resonance (SR1) from the north-south coil (a,b) and the east-west coil $(\mathrm{c}, \mathrm{d})$. Note the sunrise terminator line is visible in the east-west plots. 


\section{Searching for the Madden-Julian Oscillation}

The Madden-Julian Oscillation was originally detected as a weak periodicity in the zonal winds recorded at tropical latitudes (Madden \& Julian, 1971). Further analysis revealed it was also detectable in rainfall patterns at the equator and in the outgoing longwave radation (OLR) measurable at satellite altitude. therein).

\subsection{The Real-time Multivariate MJO (RMM) index}

As it strongly influences rainfall patterns in the tropics, monitoring the MJO is of great interest for medium to long-term weather forecasting. The Bureau of Meteorology (BoM) in Australia have developed a measure called the Real-time Multivariate MJO (RMM) index which captures the phase and amplitude of each MJO cycle. The RMM index is a daily bivariate time-series created from principal component analysis of a number of physical parameters such as OLR and zonal wind data at various altitudes (Wheeler \& Hendon, 2004). The two strength and location of the MJO. The MJO phase, which is a proxy for its location, and amplitude of each cycle can be derived from RMM1 and RMM2.

The pattern of the MJO is itself affected by other longer term phenomenon, primarily the El Niño Southern Oscillation (ENSO). In the warmer (El Niño) 230 phase the location of maximum rainfall moves eastward toward the Pacific, while during the cooler (La Niña) phase, the MJO initiates predominantly over continental Africa (Slingo et al., 1999, Zhang, 2005).

Figure 3 shows two time-longitude plots of equatorial rainfall from the Global Precipitation and Climatological Project (GPCP) V1.3 Daily Analysis Product 

$\mathrm{mm} /$ day) within the equatorial latitude band $\pm 10^{\circ}$, shown over the calendar year from January-December. The two panels to the right of the color plots show the RMM phase and amplitudes and the RMM1 and RMM2 indices from the Australian BoM. Note, the RMM phase is a discrete value between 1 and 8 plotting purposes.

In Figure 3(a), rainfall data for 2013, a La Niña year, are shown. The thin white arrows indicate some of the MJO patterns, moving eastwards over time. The pattern is repeated throughout the year. The thicker white lines indicate the start of an MJO. These coincide with the minimum of the RMM phase line (by definition, as RMM phase is 1 for the beginning of the cycle) and (often) a minimum in the amplitude. However, the direct link with rainfall occurrence and RMM1/RMM2 is more complicated - the start of each MJO is not necessarily coincident with a minimum in either index (Wheeler \& Hendon, $250 \quad 2004)$.

The time-longitude rainfall plot for 2015, an El Niño year, is shown in Figure 3(b). The most intense rainfall locations have moved eastward to around $170^{\circ} \mathrm{E}$. The white dotted lines aligned to these events show that the amplitude of the MJO is low but that the phase has not returned to its minimum value, thus indicating a very weak MJO cycle. (Note, the more numerous westward propagating patterns are Rossby waves, as described in Zhang (2005)). In terms of our analysis, the movement of the rainfall and lightning centres away from the African continent during El Niño will lead to a change in the average distance and direction of the travel path to Eskdalemuir. There may also be a fall in intensity in Europe as noted by authors such as Sátori \& Zieger (1999), Sátori et al. (2009) and Nieckarz et al. (2009).

\subsection{Periodic Analysis: Lomb-Scargle periodograms}

A direct comparison of the periodograms of the intensity and frequency of the Schumann Resonances with the RMM index is possible using the Lomb- 


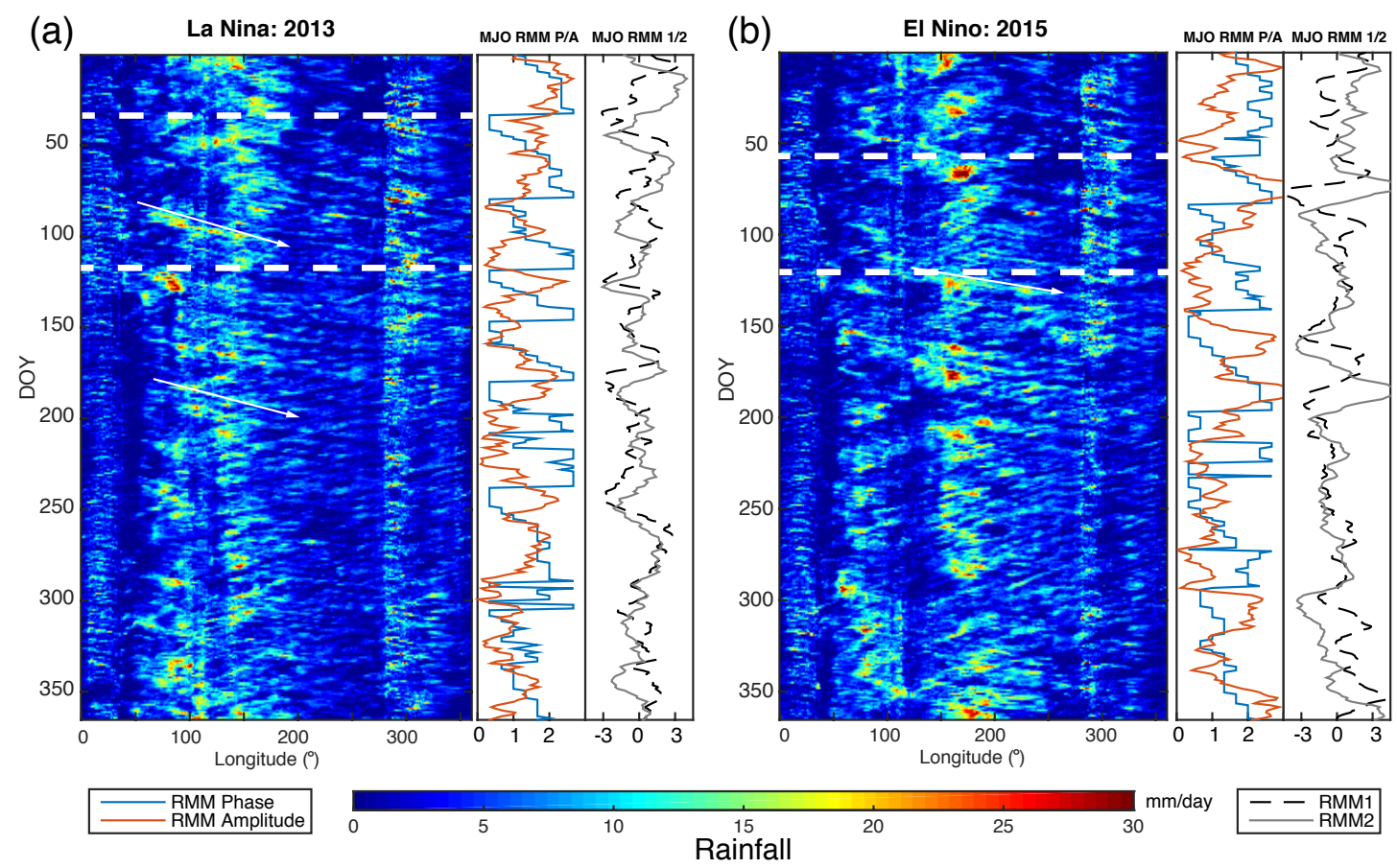

Figure 3: Time-longitude plots of daily-averaged equatorial rainfall in the latitude band $\pm 10^{\circ}$ for (a) 2013 and (b) 2015. The right-hand panels indicate RMM phase and amplitudes and the RMM1 and RMM2 indices. Dashed white lines show the initiation of MJO cycles. White arrows illustrate the eastward motion of the rainfall over time. Note the migration of the heaviest rainfall to the Pacific in El Niño years. 
Scargle periodogram. This method can account for missing data and hence is superior to an FFT, though with the inevitable loss of some accuracy (Lomb. 1976). Intensity and frequency values from the first resonance (SR1) consists of data from a total of 2221 days covering September 2012 to September 2018. We also analyzed the (complete) index values for RMM1 and RMM2 using 270 Lomb-Scargle.

Figure 4 (a) plots the power spectra, analysed using Lomb-Scargle periodograms, of the RMM index on a log-log scale, showing a broad range of maximum power between 30 to 90 days, as well as an annual peak. Figure 4 (b) and (c) show the periodograms for the intensity and frequency of SR1 275 for both induction coils. The strongest peaks are at 1 day and one year with little obvious correlation between the RMM and SR1 datasets, thus implying the MJO does not have a strong periodic influence in the SR data. There are also no obvious peaks around solar rotation periods (27 days) or shorter term components.

\subsection{Quasi-periodic Analysis: Empirical Mode Decomposition}

Empirical Mode Decomposition (EMD) is a technique for identifying nonstationary, quasi-periodic signals in time-series data. It is part of the Hilbert-Huang transform (Huang et al. 1998$)$ and consists of a sifting process which iteratively fits a spline through the minimum and maximum of the data to compute a running mean value. This mean is subtracted from the data to form the first Intrinsic Mode Function (IMF). This process is repeated until the residual becomes smaller than a predefined threshold value or contains no further minima or maxima. The technique has been applied to a large number of geophysical problems. In geomagnetism, for example, Jackson \& Mound (2010) examined 400 years of declination measurements from Paris and London to identify long period signals ( $\sim 80$ years) indicative of unknown core processes.

The EMD technique requires a complete dataset and so is sensitive to missing data values. It also tends to fail at the end points of the time-series. As the induction coil dataset is around only $95 \%$ complete, an interpolation strategy 

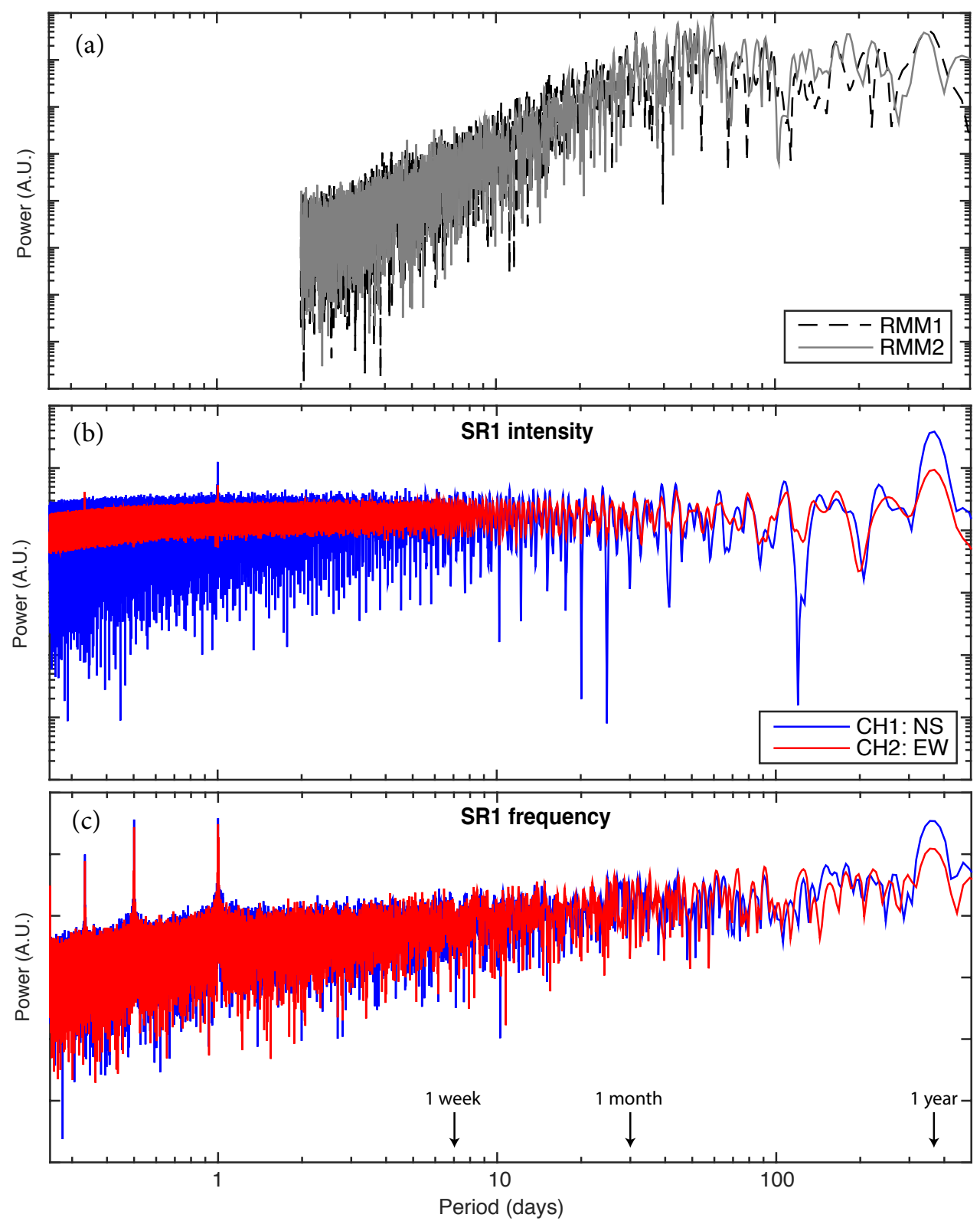

Figure 4: Lomb-Scargle periodograms of six years of data from Sep 2012 to Sep 2018 for (a) the RMM indices, (b) the intensity variation and (c) the frequency variation of the first Schumann Resonance. 
was required to fill the gaps. We also reduced the data cadence from 10-minute data points to a daily value. For each day, the 144 10-minute SR amplitude and frequency data were down-sampled to a single daily value using a finite impulse response function (rather than a simple daily average). Next, large spikes were removed from the dataset using a manually-set threshold value. Finally, linear interpolation was used to fill in the missing data to form a complete dataset. The largest gap is around 30 days due to a hardware failure, though most are less than 5 days.

We use a modified version of the EMD method from Flandrin et al. (2004) called the ensemble EMD to decompose the Schumann resonance intensity and frequency time-series. The ensemble EMD technique involves adding a small amount of random noise to each input data point in the time-series before decomposing it into the IMFs (Wu \& Huang, 2009). We used 16 ensembles and averaged the IMF outputs from each member to give a final set of IMFs for each SR parameter. This improves the robustness of the results compared to a single EMD. We also decomposed the RMM indices (RMM1, RMM2, Phase and Amplitude).

The EMD method generates up to 8 IMFs for each time series. Each IMF time-series is then passed through a peak-finding algorithm to identify the interpeak time difference. This average time difference is then ascribed to the particular IMF. IMFs with inter-peak times of less than 30 days or greater than 90 days are ignored in this analysis as being outside the range of the MJO. Using the IMFs which have periods between 30 and 90 days allows us to compare the SR1 intensity and frequency from each channel to the RMM indices in the period range that the MJO typically occupies.

Figure 5(a) shows the variation in SR1 intensity in both coil channels over the six years studied. In Figure 5(b) and (c), the relevant IMFs from the decomposition of the SR1 intensity time-series are shown (red and blue curves). The matching IMFs from the decomposition of the RMM phase curve are shown as gray lines. All curves have been normalized to their maximum absolute value to allow for easier comparison. For most of the time-series there is little direct 
correlation between the RMM IMF and the SR1 IMF. Time periods where the curves beat in phase are highlighted in pink and occur in 2013/14 and 2016 in panel (b) and 2013/14 in panel (c), primarily for the NS-orientated coil (blue curve).

The SR1 intensity IMFs are also compared to the IMFs from the decompostion of RMM1 in panels (d) and (e). The SR1 IMFs in panel (d) correlate best in the highlighted time periods, matching panels (b) and (c), but there are no obvious periods of correlation in panel (e). Finally, the Southern Oscillation Index (SOI) is plotted in panel (f). Positive values indicate a La Niña event while negative values are the El Niño phase. A strong El Niño occurred between 2014.5 and 2016.5. The highlighted regions of panels (b), (c) and (d) tend to occur in the La Niña phases.

The SR1 frequency data are plotted in Figure 6(a). The same analysis as Figure 5 is applied to the frequency time-series. The RMM phase IMFs with the most similar periods are shown in panels (b) and (c). The in-phase times (primarily with the NS orientated coil) are highlighted in pink. In panels (d) and (e) there are no apparent periods where the RMM1 IMFs and SR1 frequency IMFs coincide. Panel (f) shows the SOI with the highlighted regions from panels (b) and (c). Again, they occur around 2013/14 and 2017/18, coinciding with 345 La Niña phases.

In addition to SR1, we examined the intensity and frequency IMFs decomposed from SR2 and SR3, as well as the intensity derived from the $10 \mathrm{~Hz}$ line in the induction coil spectrograms. Overall, there was little consistency between the RMM IMFs for RMM1 and RMM2 or Amplitude and Phase, except for the SR2 Intensity with RMM1 which occasionally beats in-phase during La Niña phases. These plots for RMM1 and RMM phase are given in the Supplementary Information for reference. 

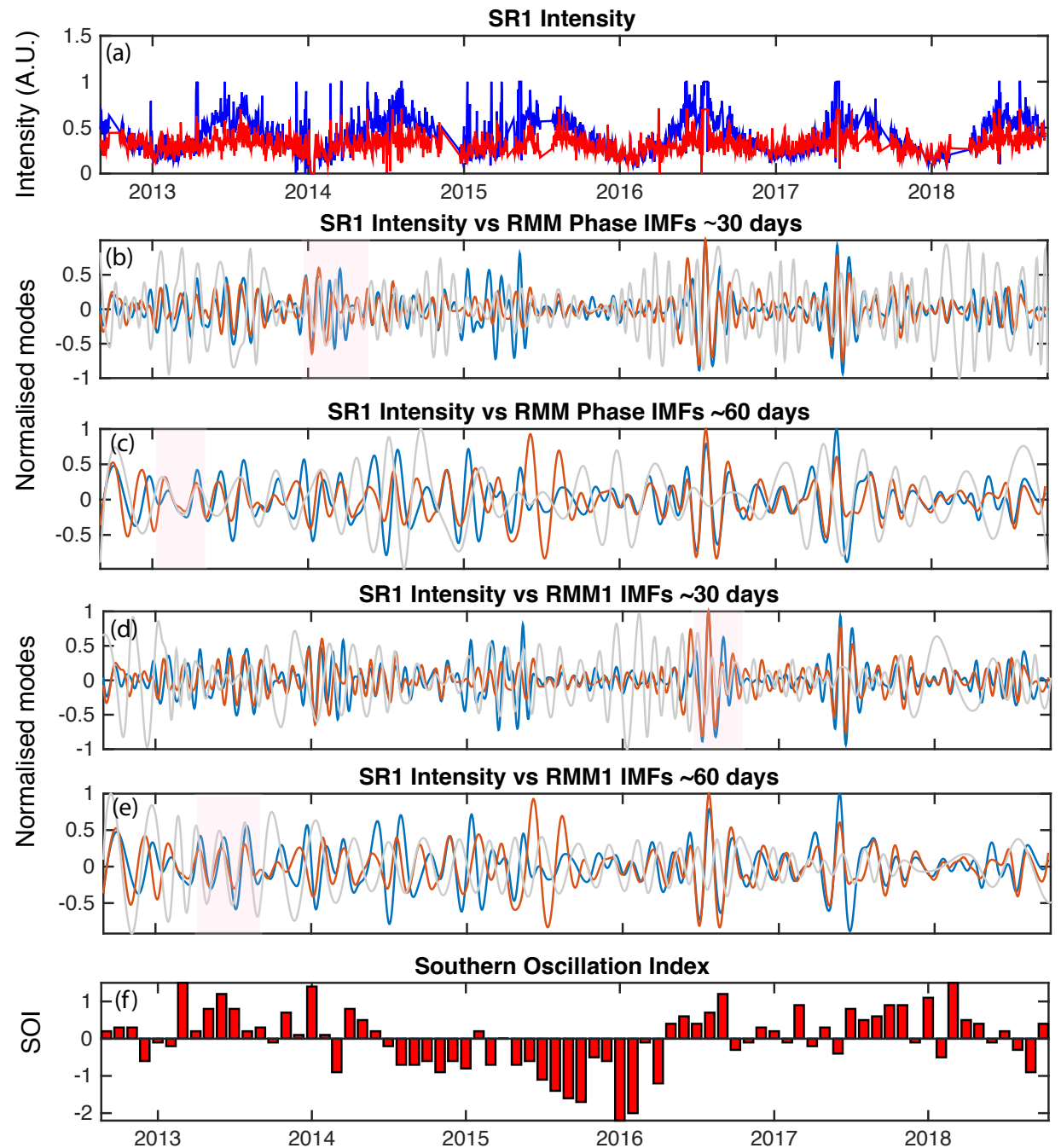

Figure 5: Comparison of the Intrinsic Mode Functions (IMF) from the decomposition of the first Schumann Resonance (SR1) intensity and the RMM phase time-series. (a) SR1 intensity in the north-south coil (blue) and east-west coil (red). (b,c) IMF curves for SR1 intensity (blue, red) and RMM phase (gray). (d,e) IMF curves for SR1 intensity (blue, red) and RMM1 (gray). (f) Southern Oscillation index; negative values indicate El Niño periods. Pink highlighted regions indicate times when the IMFs beat in-phase. 

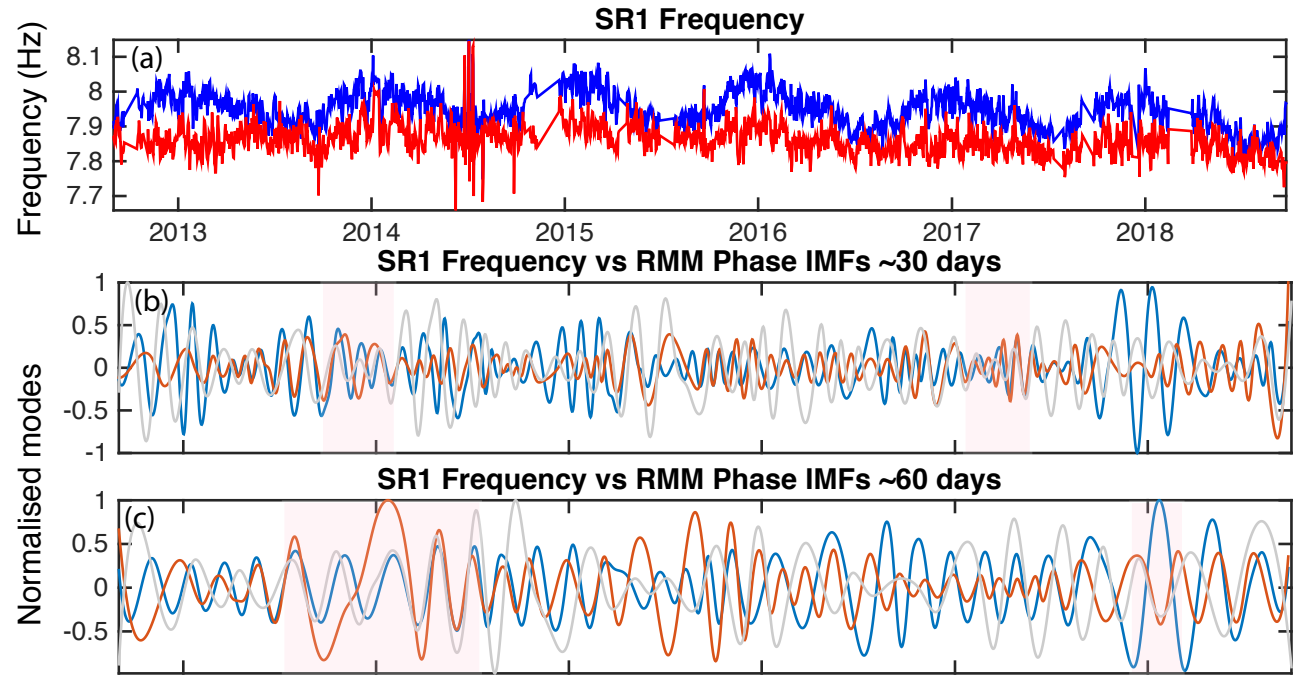

1

SR1 Frequency vs RMM1 IMFs $~ 30$ days

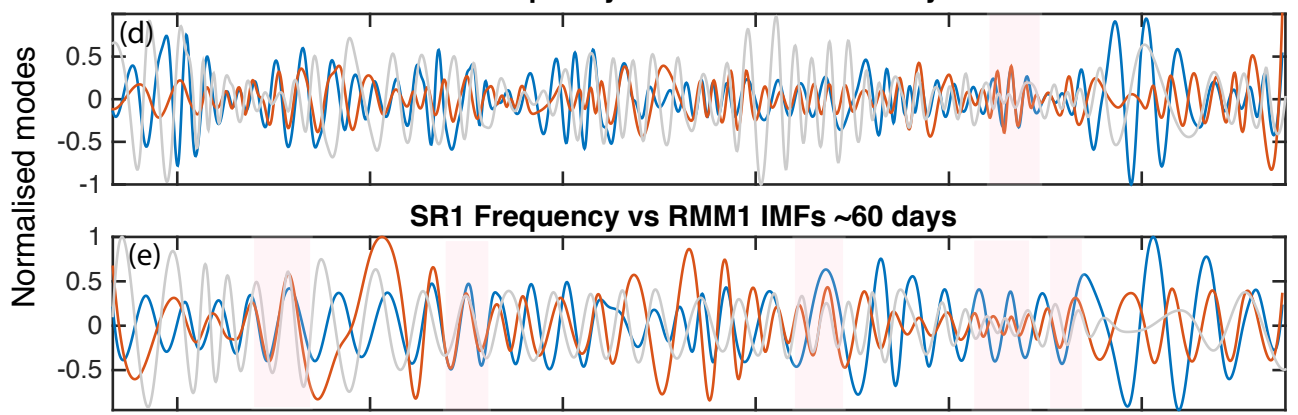

Southern Oscillation Index

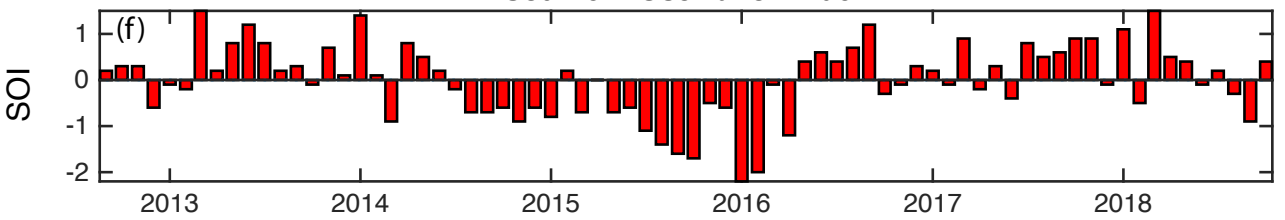

Figure 6: Comparison of the Intrinsic Mode Functions (IMF) from the decomposition of the first Schumann Resonance (SR1) frequency and the RMM phase time-series. (a) SR1 frequency in the north-south coil (blue) and east-west coil (red). (b,c) IMF curves for SR1 frequency (blue, red) and RMM phase (gray). (d,e) IMF curves for SR1 frequency (blue, red) and RMM1 (gray). (f) Southern Oscillation index; negative values indicate El Niño periods. Pink highlighted regions indicate times when the IMFs beat in-phase. 


\section{Discussion}

We have examined the intensity and frequency variations of the first three

355 duction coils at Eskdalemuir in the UK. The site is magnetically quiet and the six year dataset offers a reasonable record length to investigate seasonal and inter-annual signals. In general, the north-south coil measures larger intensity than the east-west coil but the response depends on the direction of the electromagnetic wave arrivals with respect to the source regions. Figure 1 shows that during winter the east-west coil (panel b) is slightly more energetic than the north coil for much of the day. In the summer, the opposite occurs as the north-south coil (panel c) measures higher amplitude intensities throughout the day.

We choose to search for the Madden-Julian Oscillation as a challenging signal that previous research suggests is present. Following standard practise, we extracted the time-series into ten minute blocks and computed the Welch periodogram. We then sought the intensity and peak frequency of the first three Schumann Resonances around the 8, 14 and $21 \mathrm{~Hz}$ lines. We examined these data in a series of Time-Universal Time plots, looking at the average variation over each year and the deviation away from the average. Although we noted the influence of the seasonal variation due to the change in the location and intensity of the lightning centres, there was no evidence for coherent inter-seasonal signals, suggesting they are subtle.

In Figure 2 (b), we noted that the SR1 intensity from the east-west orientated coil responds most strongly to the local afternoon (1600 UT) signals, which arise from lightning activity approximately south of the UK (i.e. the African lightning centre), while the north-south intensity (panel a) responds to lightning from India/Indonesia at around 09:00 and America later around 20:00. The intensity plots peak at the mid-summer season which coincides with the closest approach of the lightning centres. Conversely, the frequency plots show their minima at these times, illustrating the effect of the source-receiver distance on the different 
parameters (e.g. Nickolaenko \& Hayakawa, 2002).

As a proxy for the MJO, we chose the RMM index, a bivariate variable which tain years. They note, in their Section 3, that the best match between the CI and the SR was winter $1989 / 90$, which was a La Niña phase. It is noteworthy 
that no other papers, to our knowledge, have provided further evidence of the

influence of the MJO on the SR; which implies it is difficult to find.

Sátori et al. (2009) noted the unexpected reduction of the mean daily intensity of the SR at Nagycenk during El Niño conditions compared to La Niña. This is consistent with the observations at Eskdalemuir too. Satori et al. argue that the decrease during El Niño relates to the fall in the number of lightning 420 the lightning centres by around $500 \mathrm{~km}$ between ENSO phases. Thus, it may be argued that the source-receiver effect in conjunction with decrease in lightning activity in the Pacific during El Niño controls the variation of intensity of the Schumann Resonances at Eskdalemuir. During an El Niño phase the 425 lightning activity centres move eastward and lightning in the Pacific decreases. Conversely, during La Niña phases, global lightning intensity falls (though not in the Pacific) but the activity centres move westward. The MJO also initiates in different locations depending on the ENSO phase. Hence, MJO-related modulation in the SR parameters in the Eskdalemuir may be easier to distinguish conditions.

We note there are a number of limitations in this study which include the relatively short length of the dataset (six years) and the effect of noise and partial or missing data (around 5\%) on the overall analysis. The gaps affect the robustness of the EMD, in particular, though the use of the ensemble does reduce their influence. However, from the Time-UT plots, we do find the expected seasonal and annual variations in the dataset indicating the coils are sensitive to these changes. Finally, we cannot reject the null hypothesis i.e. the correlations between the MJO and the periodic variations found by the EMD are purely 440 coincidental.

We suggest that the analysis of other long term induction coil datasets at high latitude at different longitudes around the globe (e.g. Canada, Japan, New Zealand or Antarctica) could reveal whether the reliable detection of the MJO is indeed related to the source-receiver distance or perhaps dependent on judicious 
placement of the receiver relative to the motion of the lightning centres.

\section{Conclusion}

Using six years of high-quality induction coil data from Eskdalemuir Geophysical Observatory, we examine the frequency and intensity variations of the first three Schumann Resonances to search for the influence of the MaddenJulian Oscillation, an equatorial atmospheric phenomenon with a period of 3090 days.

We compare the frequency and intensity variations of the Schumann Resonances to the RMM index, a proxy for the MJO. Direct comparison between the RMM index values and the SR using Lomb periodograms reveals no common periodic signals in the 30 to 90 day frequency range. We used Empirical Mode Decomposition to extract quasi-periodic modes from each of the SR intensity and frequency, and RMM index time-series. We find that the modes of the RMM phase index and the SR intensity and frequency correlate occasionally during the cooler La Niña phases of the ENSO but not in the warmer El Niño phases. We suggest this is primarily due to the relative change in the sourcereceiver distance between Eskdalemuir and the motion of the main lightning centres during ENSO.

\section{Acknowledgements}

This research did not receive any specific grant from funding agencies in the public, commercial, or not-for-profit sectors. We acknowledge the use of the following freely available data: the daily rainfall data from the Global Precipitation Climatology Project (GPCP) at University of Maryland (http://eagle1.umd. edu/GPCP_ICDR/GPCP_Monthly.html); and the RMM indices from the Australian Government Bureau of Meteorology (www.bom.gov.au/climate/mjo/). The induction coil data, along with metadata and example MATLAB code can be found at www.bgs.ac.uk/services/NGDC/citedData/catalogue.html 
using the following DOIs to access the yearly datasets: 2012: 10.5285/6dcca52047f2-45bd-9fd1-61354450d17d; 2013: 10.5285/6c18b97e-860a-4c47-8872-bedbe953c2ab; 2014: 10.5285/397aa96c-3377-4039-a417-3111f1522214; 2015:10.5285/c09b051d94ac-4aec-a49f-34d8b4cd1327; 2016: 10.5285/aaf20915-1c3e-40f3-bc00-dafe593ff9e5 and 2017: 10.5285/25de906d-a3cd-4d64-8644-77f98aafeba6.

We thank the Geomagnetism engineering team (Tony Swan, Tim Taylor, Ted Harris and Tom Martyn) for installing, operating and maintaining the induction coil magnetometers as Eskdalemuir Geophysical Observatory. This paper is published with the permission of the Executive Director of the British Geological Survey (UKRI).

\section{References}

Anyamba, E., Williams, E., Susskind, J., Fraser-Smith, A., \& Füllekrug, M. (2000). The manifestation of the Madden-Julian Oscillation in global deep convection and in the Schumann Resonance intensity. Journal of the Atmo-

口 spheric Sciences, 57, 1029-1044. doi 10.1175/1520-0469(2000)057<1029: TMOTMJ>2.0.CO;2.

Balser, M., \& Wagner, C. (1960). Observations of Earth-ionosphere cavity resonances. Nature, 188, 638-641. doi:10.1038/188638a0

Beggan, C., \& Musur, M. (2018). Observation of Ionospheric Alfvén Resonances at 1-30 Hz and their superposition with the Schumann Resonances. Journal of Geophysical Research: S, 123, 4202-4214. doi 10.1029/2018JA025264.

De, S. S., De, B. K., Bandyopadhyay, B., Paul, S., Haldar, D. K., \& Barui, S. (2010). Studies on the shift in the frequency of the first Schumann resonance 495 a mode during a solar proton event. J Atmos Sol-Terr Phy, 72, 829-836. doi 10. $1016 / j \cdot j$ astp. 2010.04 .004 .

Flandrin, P., Rilling, G., \& Goncalves, P. (2004). Empirical mode decomposition 口 as a filter bank. IEEE Signal Processing Letters, 11, 112-114. doi 10.1109/ LSP. 2003.821662 


\section{1-9169(94)00075-Y,}

Füllekrug, M., \& Fraser-Smith, A. (1996). Further evidence for a global correlation of the Earth-ionosphere cavity resonances. Geophys. Res. Lett., 23, 505 2773-2776. doi 10.1029/96GL02612.

Greenberg, E., \& Price, C. (2007). Diurnal variations of ELF transients and background noise in the Schumann resonance band. Radio Science, 42, RS2S08. doi:10.1029/2006RS003477.

Huang, N. E., Shen, Z., Long, S. R., Wu, M. C., Shih, H. H., Zheng, Q., Yen, N.C., Tung, C. C., \& Liu, H. H. (1998). The empirical mode decomposition and the Hilbert spectrum for nonlinear and non-stationary time series analysis. Proceedings of the Royal Society of London A: Mathematical, Physical and Engineering Sciences, 454, 903-995. doi:10.1098/rspa.1998.0193.

Huffman, G. J., Adler, R. F., Morrissey, M., Bolvin, D. T., Curtis, S., Joyce, 515 R., McGavock, B., \& Susskind, J. (2001). Global precipitation at one-degree daily resolution from multi-satellite observations. J. Hydrometeor., 2, 36-50.

Jackson, L. P., \& Mound, J. E. (2010). Geomagnetic variation on decadal time scales: What can we learn from empirical mode decomposition? Geophysical Research Letters, 37, L14307. doi:10.1029/2010GL043455.

${ }_{520}$ Kułak, A., Kubisz, J., Michalec, A., Zięba, S., \& Nieckarz, Z. (2003). Solar variations in extremely low frequency propagation parameters: 2. Observations of Schumann resonances and computation of the ELF attenuation parameter. Journal of Geophysical Research: Space Physics, 108, 1-9. doi:10.1029/2002JA009305.

Lomb, N. R. (1976). Least-squares frequency analysis of unequally spaced data. Astrophysics and Space Science, 39, 447-462. 
Madden, R. A., \& Julian, P. R. (1971). Detection of a 40-50 day oscillation in the zonal wind in the tropical Pacific. Journal of the Atmospheric Sciences, 28, 702-708. doi 10.1175/1520-0469(1971)028<0702:DOADOI>2.0.C0;2.

Melnikov, A., Price, C., Sátori, G., \& Füllekrug, M. (2004). Influence of solar terminator passages on Schumann resonance parameters. Journal of Atmospheric and Solar-Terrestrial Physics, 66, 1187-1194. doi $10.1016 / j \cdot j$ astp. 2005.05.014.

Nickolaenko, A. P., \& Hayakawa, M. (2002). Resonances in the Earth-ionosphere $535 \quad$ Cavity volume 19. Springer Science \& Business Media.

Nickolaenko, A. P., Koloskov, A. V., Hayakawa, M., Yampolski, Y. M., Budanov, O. V., \& Korepanov, V. E. (2015). 11-year solar cycle in Schumann resonance data as observed in Antarctica. Sun and Geosphere, 10, 39-49.

a URL: http://newserver.stil.bas.bg/SUNGE0//00SGArhiv/SG_v10_No1_ 2015-pp-39-49.pdf.

Nieckarz, Z., Zięba, S., Kułak, A., \& Michalec, A. (2009). Study of the periodicities of lightning activity in three main thunderstorm centers based on

घ Schumann resonance measurements. Monthly W, 137, 4401-4409. doi 10 . 1175/2009MWR2920.1.

${ }_{545}$ Ondrášková, A., Kostecký, P., Ševčík, S., \& Rosenberg, L. (2007). Long-term observations of Schumann resonances at Modra observatory. Radio Science, 42, RS2S09. doi 10.1029/2006RS003478.

Ondrášková, A., Ševčík, S., \& Kostecký, P. (2011). Decrease of Schumann resonance frequencies and changes in the effective lightning areas toward the solar cycle minimum of 2008-2009. Journal of Atmospheric and Solar-Terrestrial, 73, 534-543. doi $10.1016 / j \cdot j a s t p .2010 .11 .013$.

Price, C. (2016). ELF electromagnetic waves from lightning: the Schumann resonances. Atmosphere, 7, 116. doi 10.3390/atmos7090116. 
Sátori, G. (1996). Monitoring Schumann resonances - II. Daily and seasonal frequency variations. Journal of Atmospheric and Terrestrial Physics, 58, 1483 - 1488. doi 10.1016/0021-9169(95)00146-8.

Satori, G., Szendröi, J., \& Verö, J. (1996). Monitoring Schumann resonancesI. Methodology. Journal of Atmospheric and Terrestrial Physics, 58, 14751481. doi:10.1016/0021-9169(95)00145-X.

Sátori, G., Williams, E., \& Lemperger, I. (2009). Variability of global lightning n activity on the ENSO time scale. Atmospheric Research, 91, 500-507. doi 10. $1016 / j$.atmosres.2008.06.014

Sátori, G., Williams, E., \& Mushtak, V. (2005). Response of the Earthionosphere cavity resonator to the 11-year solar cycle in X-radiation. Journal 565 ¿ of Atmospheric and Solar-Terrestrial Physics, 67, 553 - 562. doi 10.1016/j. jastp.2004.12.006.

Sátori, G., \& Zieger, B. (1999). El Niño related meridional ooscillations of a global lightning activity. Geophys. Res. Lett., 26, 1365-1368. doi 10.1029/ 1999GL900264.

${ }_{570}$ Schumann, W. O. (1952). Über die strahlungslosen Eigenschwingungen einer leitenden Kugel, die von einer Luftschicht und einer Ionosphärenhülle umgeben ist. Zeitschrift für Naturforschung A, 7, 149-154.

Sentman, D. (1995). Handbook of atmospheric electrodynamics. chapter 11. (pp. 267-295). CRC Press volume 1.

575 Slingo, J., M. Rowell, D. P., Sperber, K. R., \& Nortley, F. (1999). On the predictability of the interannual behaviour of the Madden-Julian oscillation and its relationship with El Niño. Q. J. R. Meteorol. Soc., 125, 583-609. doi:10.1002/qj . 49712555411

Welch, P. D. (1967). The use of Fast Fourier Transform for the estimation of power spectra: A method based on time averaging over short, modified 
periodograms. IEEE Transactions on Audio and Electroacoustics, 15, $70-$ 73. doi 10.1109/TAU.1967.1161901.

Wheeler, M. C., \& Hendon, H. (2004). An all-season real-time multivariate MJO index: Development of an index for monitoring and prediction.

585 \Monthly Weather Review, 132, 1917--1932. doi 10.1175/1520-0493(2004) $132<1917$ :AARMMI $>2.0 . \mathrm{CO} ; 2$.

Will (2005). Lightning and climate: A review. Atmos. Res., 76, 272-287. doi $10.1016 / j$. atmosres.2004.11.014

Williams, E., \& Sátori, G. (2007). Solar radiation-induced changes in ionospheric height and the Schumann resonance waveguide on different timescales. Radio Science, 42, RS003494. doi:10.1029/2006RS003494.

Williams, E. R. (1992). The Schumann Resonance: A global tropical thermometer. Science, 256, 1184-1187. doi 10.1126/science.256.5060.1184

Wu, Z., \& Huang, N. E. (2009). Ensemble empirical mode decomposition: A noise-assisted data analysis method. Advances in Adaptive Data Analysis, 01, 1-41. doi $10.1142 /$ S1793536909000047

Zhang, C. (2005). Madden-Julian Oscillation. Reviews of Geophysics, 43, RG2003. doi:10.1029/2004RG000158

Zieger, B., \& Sátori, G. (1999). Periodic variations of solar and tropospheric origins in Schumann resonances. In 11th International Conference on Atmospheric Electricity (pp. 701-704). NASA Conf. Publ. 